\title{
Local and Global Measurements of MGRS Rules
}

\author{
Xibei Yang 1 *, Yanqin Zhang ${ }^{2}$, Jingyu Yang ${ }^{3}$ \\ ${ }^{1}$ School of Computer Science and Engineering, Jiangsu University of Science and Technology, \\ Zhenjiang, Jiangsu, 212003, P.R. China \\ E-mail: yangxibei@hotmail.com \\ ${ }^{2}$ School of Economics, Xuzhou Institute of Technology, \\ Xuzhou, Jiangsu, 221000, P.R. China \\ E-mail:zyqxuzhou@163.com \\ ${ }^{3}$ School of Computer Science and Technology, Nanjing University of Science and Technology, \\ Nanjing, Jiangsu, 210094, P.R. China \\ E-mail: yangjy@mail.njust.edu.cn \\ Received 14 February 2012 \\ Accepted 10 August 2012
}

\begin{abstract}
Since the multigranulation rough sets (MGRS) can be considered as the compositions of multiindependent Pawlak's rough sets, the multigranulation rough set rules (MGRS rules) are then the compositions of decision rules, which are supported by multi-independent Pawlak's rough sets. To measure MGRS rules, both the local and global views are employed in this paper. In local view, the support, certainty and coverage factors are proposed to measure MGRS rules, which are support by an object; while in global view, these three factors are proposed to measure MGRS rules, which are coexisting in a decision system. The necessary conditions for these factors to achieve maximal and minimal values are also addressed. Some numerical examples are employed to substantiate the conceptual arguments.
\end{abstract}

Keywords: Certainty factor, coverage factor, decision rule, multigranulation rough set, support factor.

\section{Introduction}

Rough set theory $1,2,3,4$, proposed by Pawlak, has been demonstrated to be useful in pattern recognition, knowledge discovery ${ }^{5,6}$, decision support ${ }^{7,8}$, data mining, feature selection, medical diagnosis and so on. Pawlak's rough set, is constructed on the basis of an indiscernibility relation, which is an equivalence relation and then such model can be used to unravel decision rules from the information sys- tem with decision attributes, such system can also be referred to as decision system in many rough set literatures. It is well-known that the decision rules, which are supported by the objects in the lower approximation of the decision classes, may be referred to as certain rules; while the decision rules, which are supported by the objects in the boundary regions of the decision classes, may be referred to as possible rules.

Presently, with the rapid development of granular

${ }^{*}$ Corresponding author: No.2, Mengxi Road, School of Computer Science and Engineering, Jiangsu University of Science and Technology, Zhenjiang, Jiangsu Province, 212003, P.R. China, Tel: +86-511-84433445. 
computing ${ }^{9,10,11}$, Yao ${ }^{12}$ pointed out that Pawlak's rough set does not reflect the depth, width and $\mathrm{u}-$ niversality of granular computing in essence. With respect to Yao's triarchic theory of granular computing ${ }^{12}$, multiview, hierarchy and granular computing triangle are keys to be addressed. Multiview provides a unified framework for integrating multiple views of intelligent data analysis ${ }^{13}$. Hierarchy can not only show the relationships among different granulation structures but also reorganize the granulation structures into a multihierarchy system. Granular computing triangle summarizes pictorially the three mutually supporting perspectives on viewing, constructing and working with granular structures ${ }^{12}$.

Fortunately, to reflect the multiview of granular computing in rough set data processing, the multigranulation rough sets (MGRS) approach has been proposed by Qian et al. ${ }^{14}$. In Qian et al.'s classical MGRS, a family of the binary relations instead of a single one are used to construct the target approximations. Presently, the multigranulation approach progressing rapidly. For example, in Qian et al.'s MGRS theory, there are two different models: one is the optimistic MGRS ${ }^{15,16}$ and the other is the pessimistic MGRS ${ }^{17}$. Following Qian et al.'s work, Yang et al. generalized the MGRS into fuzzy and incomplete environments in Ref. ${ }^{18}$ and Ref. ${ }^{19}$, respectively. Xu et al. ${ }^{20}$ introduced MGRS into fuzzy tolerance approximation space. Abu-Donia ${ }^{21}$ studied the rough approximations through multi knowledge base, and then obtain the similar results to Qian et al.'s MGRS. Khan and Banerjee ${ }^{22}$ investigated the reasoning approach in multiple-source approximation systems, in which information arrives from multiple sources. Wu and Leung ${ }^{23}$ investigated the multi-scale information system, which reflects the explanation of the same problem at different scales (levels of granulations). In Refs. ${ }^{24,25}$, Qian et al. also proposed a positive approximation, which can be used to accelerate a heuristic process of attribute reduction. Since the positive approximation uses a preference ordering, which can make the granulation structure finer step by step, i.e. a finer granulation structure can be obtained by last granulation structure, then the positive approximation also re- flects the thinking of multigranulation.

It should be noticed that Qian et al.'s MGRS may be considered as the compositions of multiindependent Pawlak's rough sets. For instance, optimistic multigranulation lower approximation is the union of multi-independent Pawlak's lower approximations, pessimistic multigranulation lower approximation is the intersection of multi-independent Pawlak's lower approximations, etc. For such reason, the decision rules in terms of MGRS, i.e. MGRS rules may be regarded as the composition$\mathrm{s}$ of decision rules, which are derived from multiindependent Pawlak's rough sets.

The purpose of this paper is to measure MGRS rules. To facilitate our discussion, we first present the basic notions, which are related to rough set and MGRS in Section 2. In Section 3, by the logical connections used in MGRS, two types of MGRS rules are investigated. One is "OR" MGRS rule, while the other is "AND" MGRS rule. Three different factors are proposed to measure such two MGRS rules from the local and global views, respectively. Results are summarized in Section 4.

\section{Preliminary knowledge on rough sets}

In this section, we will review some basic concepts such as information system, Pawlak's rough set and multigranulation rough set.

\subsection{Pawlak's rough set}

Formally, an information system can be considered as a pair $I=<U, A T>$, where

- $U$ is a non-empty finite set of objects, it is called the universe;

- $A T$ is a non-empty finite set of attributes, such that $\forall a \in A T, V_{a}$ is the domain of attribute $a$.

$\forall x \in U$, let us denote by $a(x)$ the value that $x$ holds on $a(a \in A T)$. For an information system $I$, one then can describe the relationship between objects through their attributes values. With respect to a subset of attributes such that $A \subseteq A T$, an indiscernibility relation $\operatorname{IND}(A)$ may be defined as

$$
\operatorname{IND}(A)=\left\{(x, y) \in U^{2}: a(x)=a(y), \forall a \in A\right\} .
$$


The relation $\operatorname{IND}(A)$ is reflexive, symmetric and transitive, then $\operatorname{IND}(A)$ is an equivalence relation. By the indiscernibility relation $I N D(A)$, one can derive the lower and upper approximations of an arbitrary subset $X$ of $U$. They are defined as

$$
\begin{aligned}
& \underline{A}(X)=\left\{x \in U:[x]_{A} \subseteq X\right\}, \\
& \bar{A}(X)=\left\{x \in U:[x]_{A} \cap X \neq \emptyset\right\} ;
\end{aligned}
$$

where $[x]_{A}=\{y \in U:(x, y) \in \operatorname{IND}(A)\}$ is the $A$-equivalence class containing $x$. The pair $[\underline{A}(X), \bar{A}(X)]$ is referred to as the Pawlak's rough set of $X$ with respect to the set of attributes $A$.

Through lower and upper approximations, the boundary region of $X$ is

$$
B N_{A}(X)=\bar{A}(X)-\underline{A}(X) .
$$

\subsection{MGRS}

The MGRS is different from Pawlak's rough set model because the former is constructed on the basis of a family of indiscernibility relations instead of a single one.

In Qian et al.'s MGRS theory, two different models have been defined. The first one is optimistic MGRS, the second one is pessimistic MGRS.

\subsubsection{Optimistic MGRS}

In Qian et al.'s optimistic MGRS, the target is approximated through a family of the indiscernibility relations. In lower approximation, the word "optimistic" is used to express the idea that in multi independent indiscernibility relations, we need only at least one of the indiscernibility relations to satisfy with the inclusion condition between equivalence class and target. The upper approximation of optimistic MGRS is defined by the complement of the lower approximation.

Definition 1. ${ }^{15,16}$ Let $I$ be an information system, in which $A_{1}, A_{2}, \cdots, A_{m} \subseteq A T$, then $\forall X \subseteq U$, the optimistic multigranulation lower and upper approximations are denoted by $\sum_{i=1}^{m} A_{i}{ }^{O}(X)$ and ${\overline{\sum_{i=1}^{m} A_{i}}}^{O}(X)$, respectively,

$$
\begin{aligned}
& \sum_{i=1}^{m} A_{i}(X)=\left\{x \in U:[x]_{A_{1}} \subseteq X \vee \cdots \vee[x]_{A_{m}} \subseteq X\right\} ; \\
& \overline{\sum_{i=1}^{m} A_{i}}(X)=\sim \sum_{i=1}^{m} A_{i} O
\end{aligned}
$$

where $[x]_{A_{i}}(1 \leqslant i \leqslant m)$ is the equivalence class of $x$ in terms of set of attributes $A_{i}, \sim X$ is the complement of set $X$.

By the lower and upper approximations $\sum_{i=1}^{m} A_{i}{ }^{O}(X)$ and ${\overline{\sum_{i=1}^{m} A_{i}}}^{O}(X)$, the optimistic multigranulation boundary region of $X$ is

$$
B N_{\sum_{i=1}^{m} A_{i}}^{O}(X)=\overline{\sum_{i=1}^{m} A_{i}}(X)-\sum_{i=1}^{m} A_{i}^{O}(X) .
$$

Theorem 1. Let I be an information system, in which $A_{1}, A_{2}, \cdots, A_{m} \subseteq A T$, then $\forall X \subseteq U$, we have

$$
\overline{\sum_{i=1}^{m} A_{i}}(X)=\left\{x \in U:[x]_{A_{1}} \cap X \neq \emptyset \wedge \cdots \wedge[x]_{A_{m}} \cap X \neq \emptyset\right\} \text {. }
$$

Proof. By Definition 1, we have

$$
\begin{aligned}
x \in{\overline{\sum_{i=1}^{m} A_{i}}}^{O}(X) & \Leftrightarrow x \notin \sum_{i=1}^{m} A_{i}(\sim X) \\
& \Leftrightarrow[x]_{A_{1}} \nsubseteq(\sim X) \wedge \cdots \wedge[x]_{A_{m}} \nsubseteq(\sim X) \\
& \Leftrightarrow[x]_{A_{1}} \cap X \neq \emptyset \wedge \cdots \wedge[x]_{A_{m}} \cap X \neq \emptyset .
\end{aligned}
$$

By Theorem 1, we can see that though the optimistic multigranulation upper approximation is defined by the complement of the optimistic multigranulation lower approximation, it can also be considered as a set, in which objects have non-empty intersection with the target in terms of each indiscernibility relation.

\subsubsection{Pessimistic MGRS}

In Qian et al.'s pessimistic MGRS, the target is still approximated through a family of the indiscernibil- 
ity relations. However, it is different from the optimistic case. In lower approximation, the word "pessimistic" is used to express the idea that in multi independent indiscernibility relations, we need all of the indiscernibility relations to satisfy with the inclusion condition between equivalence class and target. The upper approximation of pessimistic multigranulation rough set is also defined by the complement of the pessimistic multigranulation lower approximation.

Definition 2. ${ }^{17}$ Let $I$ be an information system, in which $A_{1}, A_{2}, \cdots, A_{m} \subseteq A T$, then $\forall X \subseteq$ $U$, the pessimistic multigranulation lower and upper approximations are denoted by $\sum_{i=1}^{m} A_{i}^{P}(X)$ and ${\overline{\sum_{i=1}^{m} A_{i}}}^{P}(X)$, respectively,

$$
\begin{aligned}
& \sum_{i=1}^{m} A_{i}(X)=\left\{x \in U:[x]_{A_{1}} \subseteq X \wedge \cdots \wedge[x]_{A_{m}} \subseteq X\right\} ; \\
& {\overline{\sum_{i=1}^{m} A_{i}}}^{P}(X)=\sim \sum_{i=1}^{m} A_{i}{ }^{P}(\sim X) .
\end{aligned}
$$

By the lower and upper approximations $\sum_{i=1}^{m} A_{i}^{P}(X)$ and ${\overline{\sum_{i=1}^{m} A_{i}}}^{P}(X)$, the pessimistic multigranulation boundary region of $X$ is

$$
B N_{\sum_{i=1}^{m} A_{i}}^{P}(X)={\overline{\sum_{i=1}^{m} A_{i}}}^{P}(X)-\underline{\underline{\sum_{i=1}^{m} A_{i}}}{ }^{P}(X) .
$$

Theorem 2. Let I be an information system, in which $A_{1}, A_{2}, \cdots, A_{m} \subseteq A T$, then $\forall X \subseteq U$, we have

$$
{\overline{\sum_{i=1}^{m} A_{i}}}^{P}(X)=\left\{x \in U:[x]_{A_{1}} \cap X \neq \emptyset \vee \cdots \vee[x]_{A_{m}} \cap X \neq \emptyset\right\} .
$$

Proof. The proof of Theorem 2 is similar to the proof of Theorem 1.

Different from the upper approximation of optimistic MGRS, the upper approximation of pessimistic MGRS is represented as a set, in which objects have non-empty intersection with the target in terms of at least one of the indiscernibility relations.
Theorem 3. Let I be an information system, in which $A_{1}, A_{2}, \cdots, A_{m} \subseteq A T$, then we have

$$
\begin{aligned}
& \sum_{i=1}^{m} A_{i}{ }^{O}(X)=\bigcup_{i=1}^{m} \underline{A_{i}}(X) ; \\
& \overline{\sum_{i=1}^{m} A_{i}}(X)=\bigcap_{i=1}^{m} \overline{A_{i}}(X) ; \\
& \overline{\sum_{i=1}^{m} A_{i}}(X)=\bigcap_{i=1}^{m} \underline{A_{i}}(X) ; \\
& \overline{\sum_{i=1}^{m} A_{i}}(X)=\bigcup_{i=1}^{m} \overline{A_{i}}(X) .
\end{aligned}
$$

Proof. It can be derived directly from Definition 1, Theorem 1, Definition 2 and Theorem 2.

\subsection{Multigranulation rough memberships}

In Pawlak's rough set model, there is a direct relationship between rough approximation and the membership such that

$$
\begin{array}{r}
\mu_{X}^{A}(x)=1 \Leftrightarrow x \in \underline{A}(X) ; \\
0<\mu_{X}^{A}(x) \leqslant 1 \Leftrightarrow x \in \bar{A}(X) .
\end{array}
$$

where $\mu_{X}^{A}(x)=\frac{\left|\mu_{A}(x) \cap X\right|}{\left|\mu_{A}(x)\right|},|X|$ denotes the cardinal number of set $X$.

It should be noticed that since more than one equivalence relations are used in MGRS approach, the re-definition of the rough membership has become a necessity.

Definition 3. Let $I$ be an information system, in which $A_{1}, A_{2}, \cdots, A_{m} \subseteq A T, \forall X \subseteq U$, the maximal and minimal rough memberships of $x$ in $X$ are denoted by $\mu_{X_{M A X}}^{\sum_{i=1}^{m} A_{i}}(x)$ and $\mu_{X_{M I N}}^{\sum_{i=1}^{m} A_{i}}(x)$, respectively, where

$$
\begin{gathered}
\mu_{X_{M A X}}^{\sum_{i=1}^{m} A_{i}}(x)=\max _{i=1}^{m} \mu_{X}^{A_{i}}(x) ; \\
\mu_{X_{M I N}}^{\sum_{i=1}^{m} A_{i}}(x)=\min _{i=1}^{m} \mu_{X}^{A_{i}}(x) .
\end{gathered}
$$

Theorem 4. Let I be an information system, in which $A_{1}, A_{2}, \cdots, A_{m} \subseteq A T, \forall X \subseteq U$, we have 
1. $\mu_{X_{M A X} \sum_{i=1}^{m} A_{i}}(x)=1 \Leftrightarrow x \in \underline{\sum_{i=1}^{m} A_{i}^{O}(X) ;}$

2. $0<\mu_{X_{M I N}}^{\sum_{i=1}^{m} A_{i}}(x) \leqslant 1 \Leftrightarrow x \in \overline{\sum_{i=1}^{m} A_{i}}(X)$

3. $\mu_{X_{M I N}}^{\sum_{i=1}^{m} A_{i}}(x)=1 \Leftrightarrow x \in \sum_{i=1}^{m} A_{i}^{P}(X)$;

4. $0<\mu_{X_{M A X}}^{\sum_{i=1}^{m} A_{i}}(x) \leqslant 1 \Leftrightarrow x \in \overline{\sum_{i=1}^{m} A_{i}}(X)$.

Proof. We only prove 1, others can be proved analogously.

$$
\begin{aligned}
\mu_{X_{M A X}}^{\sum_{i=1}^{m} A_{i}}(x)=1 & \Leftrightarrow \max _{i=1}^{m} \mu_{X}^{A_{i}}(x)=1 \\
& \Leftrightarrow \quad \exists i \in\{1,2, \cdots, m\} \text { s.t. }[x]_{A_{i}} \subseteq X \\
& \Leftrightarrow x \in \underline{\sum_{i=1}^{m} A_{i}}(X) .
\end{aligned}
$$

\section{MGRS rules}

\subsection{Compositions of rules}

The end result of rough set model is a representation of the information contained in the data system considered in terms of "if... then..." decision rules. The decision rules can be generated from the decision system in the rough set approach. A decision system is an information system such that $I=$ $(U, A T \cup D)$, in which $A T$ is the set of conditional attributes, while $D$ is the set of decisional attributes. In this paper, to simplify our discussion, we only consider one decision attribute $d$ and then the decision system can be represented by $I=(U, A T \cup\{d\})$. Generally speaking, we may assume that such decision attribute determines a partition on the universe of discourse, i.e $U / \operatorname{IND}(\{d\})=\left\{X_{1}, X_{2}, \cdots, X_{k}\right\}$.

Following Pawlak's rough set model, it is wellknown that the decision rules can be derived such that

$a_{1}(y)=v_{1} \wedge a_{2}(y)=v_{2} \wedge \cdots \wedge a_{s}(y)=v_{s} \rightarrow y \in X_{j}$, in which $\left\{a_{1}, a_{2}, \cdots, a_{s}\right\}=A T, X_{j} \in U / I N D(\{d\})$.

Given an object $x \in U$, if $a_{1}(x)=v_{1}, a_{2}(x)=$ $v_{2}, \cdots, a_{s}(x)=v_{s}$, then the above decision rule is said to be supported by object $x$, thus, such rule can be denoted by

$$
y \in[x]_{A T} \rightarrow y \in X_{j}
$$

Moreover, if $x \in \underline{A T}\left(X_{j}\right)$, then $y \in[x]_{A T} \rightarrow y \in X_{j}$ is a certain rule; if $x \in B N_{A T}\left(X_{j}\right)$, then $y \in[x]_{A T} \rightarrow$ $y \in X_{j}$ is a possible rule.

Through Theorem 3, it is not difficult to observe that MGRS can be considered as the compositions of multi-independent Pawlak's rough sets. For instance, the optimistic multigranulation lower approximation is the union of $m$ Pawlak's lower approximations. Therefore, the MGRS rules derived may be regarded as the compositions of multi-independent rules, which are generated from Pawlak's rough sets. By the logical connectives used in MGRS, i.e. $\vee$ and $\wedge$, the multi-independent rules can be fused through the words "OR" and "AND", respectively.

For instance, take for instance $x \in \sum_{i=1}^{m} A_{i}{ }^{O}(X)$, by Theorem 3, we know that $x \in \overline{A_{1}(X)}$ or $x \in$ $A_{2}(X), \cdots$, or $x \in A_{m}(X)$. Therefore, we can derive the following MGRS rules:

$$
\begin{aligned}
& y \in[x]_{A_{1}} \rightarrow y \in X \text { or } \\
& y \in[x]_{A_{2}} \rightarrow y \in X \text { or } \\
& \vdots \\
& y \in[x]_{A_{m}} \rightarrow y \in X
\end{aligned}
$$

The above MGRS rules is denoted by $\bigvee_{i=1}^{m}[x]_{A_{i}} \rightarrow X$ in this paper.

Similarity, if the word "AND" is considered, the MGRS rules $\bigwedge_{i=1}^{m}[x]_{A_{i}} \rightarrow X$ is

$$
\begin{aligned}
& y \in[x]_{A_{1}} \rightarrow y \in X \text { and } \\
& y \in[x]_{A_{2}} \rightarrow y \in X \text { and } \\
& \vdots \\
& y \in[x]_{A_{m}} \rightarrow y \in X
\end{aligned}
$$




\subsection{Local measuring of MGRS rules}

In recent years, how to evaluate the decision performance of a decision rule has become a very important issue in rough set theory. With respect to different requirements, many different measurements have been proposed. In this section, we will generalize three widely used measurements, i.e. support, certainty and coverage factors into MGRS rules we mentioned in the last subsection.

Definition 4. Let $I$ be a decision system, in which $A_{1}, A_{2}, \cdots, A_{m} \subseteq A T, \forall x \in U$ and $\forall X_{j} \in$ $U / I N D(\{d\})$,

1. the support factor of $\bigvee_{i=1}^{m}[x]_{A_{i}} \rightarrow X_{j}$ is

$$
\operatorname{Supp}\left(\bigvee_{i=1}^{m}[x]_{A_{i}} \rightarrow X_{k}\right)=\max \left\{\frac{\left|[x]_{A_{i}} \cap X_{j}\right|}{|U|}: i=1, \cdots, m\right\} ;
$$

2. the support factor of $\bigwedge_{i=1}^{m}[x]_{A_{i}} \rightarrow X_{j}$ is

$$
\operatorname{Supp}\left(\bigwedge_{i=1}^{m}[x]_{A_{i}} \rightarrow X_{k}\right)=\min \left\{\frac{\left|[x]_{A_{i}} \cap X_{j}\right|}{|U|}: i=1, \cdots, m\right\}
$$

3. the certainty factor of $\bigvee_{i=1}^{m}[x]_{A_{i}} \rightarrow X_{j}$ is

$$
\operatorname{Cer}\left(\bigvee_{i=1}^{m}[x]_{A_{i}} \rightarrow X_{k}\right)=\max \left\{\frac{\left|[x]_{A_{i}} \cap X_{j}\right|}{\left|[x]_{A_{i}}\right|}: i=1, \cdots, m\right\}
$$

4. the certainty factor of $\bigwedge_{i=1}^{m}[x]_{A_{i}} \rightarrow X_{j}$ is

$$
\operatorname{Cer}\left(\bigwedge_{i=1}^{m}[x]_{A_{i}} \rightarrow X_{k}\right)=\min \left\{\frac{\left|[x]_{A_{i}} \cap X_{j}\right|}{\left|[x]_{A_{i}}\right|}: i=1, \cdots, m\right\} ;
$$

5. the coverage factor of $\bigvee_{i=1}^{m}[x]_{A_{i}} \rightarrow X_{j}$ is

$$
\operatorname{Cov}\left(\bigvee_{i=1}^{m}[x]_{A_{i}} \rightarrow X_{k}\right)=\max \left\{\frac{\left|[x]_{A_{i}} \cap X_{j}\right|}{\left|X_{j}\right|}: i=1, \cdots, m\right\} ;
$$

6. the coverage factor of $\bigwedge_{i=1}^{m}[x]_{A_{i}} \rightarrow X_{j}$ is
Obviously, $\quad \frac{\left|[x]_{A_{i}} \cap X_{j}\right|}{|U|}, \quad \frac{\left|[x]_{A_{i}} \cap X_{j}\right|}{\left|[x]_{A_{i}}\right|} \quad$ and $\frac{\left|[x]_{A_{i}} \cap X_{j}\right|}{\left|X_{j}\right|}$ are support, certainty and coverage factors, which have been used to measure the decision rule $y \in[x]_{A_{i}} \rightarrow y \in X_{j}$.

In Definition 4, since families of these factors are considered, then the "max" and "min" operators are defined to measure the compositions of decision rules. Obviously, if only one subset of the attributes, e.g. $A_{i}$, is considered, then these factors will degenerate into the factors to measure the single decision rule $y \in[x]_{A_{i}} \rightarrow y \in X_{j}$.

By Definition 4, it is not difficult to observe that

$$
\begin{array}{r}
\operatorname{Supp}\left(\bigvee_{i=1}^{m}[x]_{A_{i}} \rightarrow X_{j}\right) \in[0,1], \\
\operatorname{Supp}\left(\bigwedge_{i=1}^{m}[x]_{A_{i}} \rightarrow X_{j}\right) \in[0,1], \\
\operatorname{Cer}\left(\bigvee_{i=1}^{m}[x]_{A_{i}} \rightarrow X_{j}\right) \in[0,1], \\
\operatorname{Cer}\left(\bigwedge_{i=1}^{m}[x]_{A_{i}} \rightarrow X_{j}\right) \in[0,1], \\
\operatorname{Cov}\left(\bigvee_{i=1}^{m}[x]_{A_{i}} \rightarrow X_{j}\right) \in[0,1], \\
\operatorname{Cov}\left(\bigwedge_{i=1}^{m}[x]_{A_{i}} \rightarrow X_{j}\right) \in[0,1] .
\end{array}
$$

Moreover, since $0 \leqslant \operatorname{Cer}\left(\bigvee_{i=1}^{m}[x]_{A_{i}} \rightarrow X_{j}\right) \leqslant$ 1 and $0 \leqslant \operatorname{Cer}\left(\bigwedge_{i=1}^{m}[x]_{A_{i}} \rightarrow X_{j}\right) \leqslant 1$, then MGRS rules $\bigvee_{i=1}^{m}[x]_{A_{i}} \rightarrow X_{j}$ is referred to as certain (possible) if and only if $\operatorname{Cer}\left(\bigvee_{i=1}^{m}[x]_{A_{i}} \rightarrow\right.$ $\left.; X_{j}\right)=1\left(\operatorname{Cer}\left(\bigvee_{i=1}^{m}[x]_{A_{i}} \rightarrow X_{j}\right)>0\right)$, the MGRS rules $\bigwedge_{i=1}^{m}[x]_{A_{i}} \rightarrow X_{j}$ is referred to as certain (possible) if and only if $\operatorname{Cer}\left(\bigwedge_{i=1}^{m}[x]_{A_{i}} \rightarrow X_{j}\right)=$ $1\left(\operatorname{Cer}\left(\bigwedge_{i=1}^{m}[x]_{A_{i}} \rightarrow X_{j}\right)>0\right)$.

$$
\operatorname{Cov}\left(\bigwedge_{i=1}^{m}[x]_{A_{i}} \rightarrow X_{k}\right)=\min \left\{\frac{\left|[x]_{A_{i}} \cap X_{j}\right|}{\left|X_{j}\right|}: i=1, \cdots, m\right\} .
$$

Theorem 5. Let I be a decision system, in which $A_{1}, A_{2}, \cdots, A_{m} \subseteq A T, \forall x \in U$ and $\forall X_{j} \in$ 
$U / I N D(\{d\})$, we have

$$
\begin{gathered}
\operatorname{Supp}\left(\bigvee_{i=1}^{m}[x]_{A_{i}} \rightarrow X_{k}\right)>0 \Leftrightarrow x \in \overline{\sum_{i=1}^{\bar{m} A_{i}} P}\left(X_{j}\right) \\
\operatorname{Supp}\left(\bigwedge_{i=1}^{m}[x]_{A_{i}} \rightarrow X_{k}\right)>0 \Leftrightarrow x \in \overline{\sum_{i=1}^{m} A_{i}}\left(X_{j}\right) .
\end{gathered}
$$

Proof. We only prove (1), the proof of (2) is similar to the proof of (1).

$$
\begin{aligned}
& \operatorname{Supp}\left(\bigvee_{i=1}^{m}[x]_{A_{i}} \rightarrow X_{j}\right)>0 \\
\Leftrightarrow & \max \left\{\frac{\left|[x]_{A_{i}} \cap X_{j}\right|}{|U|}: i=1,2, \cdots, m\right\}>0 \\
\Leftrightarrow & \exists i \in\{1,2, \cdots, m\},[x]_{A_{i}} \cap X_{j} \neq \emptyset \\
\Leftrightarrow & \quad x \in \sum_{i=1}^{m} A_{i}\left(X_{j}\right) .
\end{aligned}
$$

Theorem 5 shows the relationships between support factors of MGRS rules and multigranulation upper approximations.

Theorem 6. Let $I$ be a decision system, in which $A_{1}, A_{2}, \cdots, A_{m} \subseteq A T, \forall x \in U$ and $\forall X_{j} \in$ $U / \operatorname{IND}(\{d\})$, we have

$$
\begin{aligned}
& \operatorname{Cer}\left(\bigvee_{i=1}^{m}[x]_{A_{i}} \rightarrow X_{j}\right)=1 \Leftrightarrow x \in \sum_{i=1}^{m} A_{i}\left(X_{j}\right) ; \\
& \operatorname{Cer}\left(\bigwedge_{i=1}^{m}[x]_{A_{i}} \rightarrow X_{j}\right)=1 \Leftrightarrow x \in \overline{\sum_{i=1}^{m} A_{i}}\left(X_{j}\right) ; \\
& \operatorname{Cer}\left(\bigvee_{i=1}^{m}[x]_{A_{i}} \rightarrow X_{j}\right)>0 \Leftrightarrow x \in \overline{\sum_{i=1}^{m} A_{i}}\left(X_{j}\right) ; \\
& \operatorname{Cer}\left(\bigwedge_{i=1}^{m}[x]_{A_{i}} \rightarrow X_{j}\right)>0 \Leftrightarrow x \in \overline{\sum_{i=1}^{m} A_{i}}\left(X_{j}\right) .
\end{aligned}
$$

Proof. We only prove (3), the proof of (4), (5) and (6) are similar to the proof of (3). $\forall i \in\{1,2, \cdots, m\}, \quad$ we know that $0 \leqslant$ $\frac{\left|[x]_{A_{i}} \cap X_{j}\right|}{\left|[x]_{A_{i}}\right|} \leqslant 1$ holds. Therefore,

$$
\begin{aligned}
& \operatorname{Cer}\left(\bigvee_{i=1}^{m}[x]_{A_{i}} \rightarrow X_{j}\right)=1 \\
& \Leftrightarrow \exists i \in\{1,2, \cdots, m\}, \frac{\left|[x]_{A_{i}} \cap X_{j}\right|}{\left|[x]_{A_{i}}\right|}=1 \\
& \Leftrightarrow \quad \exists i \in\{1,2, \cdots, m\},[x]_{A_{i}} \subseteq X_{j} \\
& \Leftrightarrow \quad x \in \sum_{i=1}^{m} A_{i}\left(X_{j}\right) .
\end{aligned}
$$

Theorem 6 shows the relationships between certainty factors of MGRS rules and multigranulation approximations. The details are: $\bigvee_{i=1}^{m}[x]_{A_{i}} \rightarrow X_{j}$ is certain if and only if it is supported by the object in optimistic multigranulation lower approximation of $X_{j} ; \bigwedge_{i=1}^{m}[x]_{A_{i}} \rightarrow X_{j}$ is certain if and only if it is supported by the object in pessimistic multigranulation lower approximation of $X_{j} ; \bigvee_{i=1}^{m}[x]_{A_{i}} \rightarrow X_{j}$ is possible if and only if it is supported by the object in pessimistic multigranulation upper approximation of $X_{j} ; \bigwedge_{i=1}^{m}[x]_{A_{i}} \rightarrow X_{j}$ is possible if and only if it is supported by the object in optimistic multigranulation upper approximation of $X_{j}$.

Theorem 7. Let $I$ be a decision system, in which $A_{1}, A_{2}, \cdots, A_{m} \subseteq A T, \forall x \in U$ and $\forall X_{j} \in$ $U / \operatorname{IND}(\{d\})$, we have

$$
\begin{gathered}
\operatorname{Cov}\left(\bigvee_{i=1}^{m}[x]_{A_{i}} \rightarrow X_{j}\right)>0 \Leftrightarrow x \in \overline{\sum_{i=1}^{m} A_{i}}\left(X_{j}\right) ; \\
\operatorname{Cov}\left(\bigwedge_{i=1}^{m}[x]_{A_{i}} \rightarrow X_{j}\right)>0 \Leftrightarrow x \in \overline{\sum_{i=1}^{m} A_{i}\left(X_{j}\right) .}
\end{gathered}
$$

Proof. The proof of Theorem 7 is similar to the proof of Theorem 5.

Theorem 7 shows the relationships between coverage factors of MGRS rules and multigranulation upper approximations. 


\subsection{Illustrative example}

Let us use an illustrative example to explain MGRS rules we mentioned above. Suppose that the director of the school must give a global evaluation to some students. This evaluation should be based on the level in Mathematics, Physics and Literature. The director gave the examples of evaluation as shown in Table 1. The example contains eight students described by means of four attributes:

- $a_{1} \Rightarrow$ level in Mathematics (conditional attribute),

- $a_{2} \Rightarrow$ level in Physics (conditional attribute),

- $a_{3} \Rightarrow$ level of Literature (conditional attribute),

- $d \Rightarrow$ global evaluation (decisional attribute).

Table 1. An example of students' evaluations.

\begin{tabular}{ccccc}
\hline$U$ & $a_{1}$ & $a_{2}$ & $a_{3}$ & $d$ \\
\hline$x_{1}$ & 2 & 3 & 2 & Bad \\
$x_{2}$ & 5 & 1 & 3 & Medium \\
$x_{3}$ & 5 & 2 & 4 & Bad \\
$x_{4}$ & 3 & 5 & 3 & Good \\
$x_{5}$ & 1 & 3 & 4 & Bad \\
$x_{6}$ & 2 & 5 & 3 & Medium \\
$x_{7}$ & 3 & 1 & 2 & Bad \\
$x_{8}$ & 2 & 1 & 2 & Medium \\
\hline
\end{tabular}

By the decision attribute, the universe is partitioned into subsets such that $U / \operatorname{IND}(\{d\})=\{$ Bad, Medium, Good $\}=$ $\left\{\left\{x_{1}, x_{3}, x_{5}, x_{7}\right\},\left\{x_{2}, x_{6}, x_{8}\right\},\left\{x_{4}\right\}\right\}$. If each attribute is employed to construct an equivalence relation, we then obtain the following multigranulation approximations:

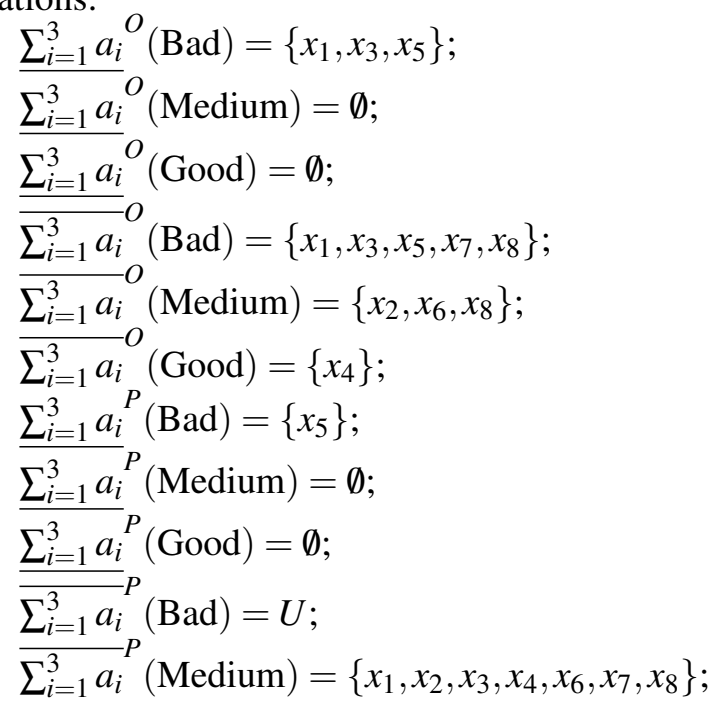

${\overline{\sum_{i=1}^{3} a_{i}}}^{P}($ Good $)=\left\{x_{2}, x_{4}, x_{6}, x_{7}\right\}$.

By Theorem 6, we know that the MGRS rules with certainty factors greater than 0 , are supported by objects in multigranulation approximations. Therefore, it is not difficult to derive the following MGRS rules:

"OR" MGRS rules:

1. $\bigvee_{i=1}^{3}\left[x_{1}\right]_{a_{i}} \rightarrow \mathrm{Bad}: a_{1}(x)=2 \rightarrow d(x)=\mathrm{Bad}$ or $a_{2}(x)=3 \rightarrow d(x)=$ Bad or $a_{3}(x)=$ $2 \rightarrow d(x)=\operatorname{Bad} / /$ supported by $x_{1} \in$ $\underline{\sum_{i=1}^{3} a_{i}}{ }^{O}(\mathrm{Bad}),{\overline{\sum_{i=1}^{3} a_{i}}}^{P}(\mathrm{Bad})$

2. $\bigvee_{i=1}^{3}\left[x_{2}\right]_{a_{i}} \rightarrow \mathrm{Bad}: a_{1}(x)=5 \rightarrow d(x)=\mathrm{Bad}$ or $a_{2}(x)=1 \rightarrow d(x)=$ Bad or $a_{3}(x)=3 \rightarrow$ $d(x)=\mathrm{Bad} / /$ supported by $x_{2} \in{\overline{\sum_{i=1}^{3} a_{i}}}^{P}(\mathrm{Bad})$

3. $\bigvee_{i=1}^{3}\left[x_{3}\right]_{a_{i}} \rightarrow \mathrm{Bad}: a_{1}(x)=5 \rightarrow d(x)=\mathrm{Bad}$ or $a_{2}(x)=2 \rightarrow d(x)=$ Bad or $a_{3}(x)=$ $4 \rightarrow d(x)=\operatorname{Bad} / /$ supported by $x_{3} \in$ $\underline{\sum_{i=1}^{3} a_{i}{ }^{O}}(\mathrm{Bad}),{\overline{\sum_{i=1}^{3} a_{i}}}^{P}(\mathrm{Bad})$

4. $\bigvee_{i=1}^{3}\left[x_{4}\right]_{a_{i}} \rightarrow \mathrm{Bad}: a_{1}(x)=3 \rightarrow d(x)=\mathrm{Bad}$ or $a_{2}(x)=5 \rightarrow d(x)=$ Bad or $a_{3}(x)=3 \rightarrow$ $d(x)=\mathrm{Bad} / /$ supported by $x_{4} \in{\overline{\sum_{i=1}^{3} a_{i}}}^{P}(\mathrm{Bad})$

5. $\bigvee_{i=1}^{3}\left[x_{5}\right]_{a_{i}} \rightarrow \mathrm{Bad}: a_{1}(x)=1 \rightarrow d(x)=\mathrm{Bad}$ or $a_{2}(x)=3 \rightarrow d(x)=$ Bad or $a_{3}(x)=$ $4 \rightarrow d(x)=\mathrm{Bad} / /$ supported by $x_{5} \in$ $\sum_{i=1}^{3} a_{i}{ }^{O}(\mathrm{Bad}),{\overline{\sum_{i=1}^{3} a_{i}}}^{P}(\mathrm{Bad})$

6. $\bigvee_{i=1}^{3}\left[x_{6}\right]_{a_{i}} \rightarrow \mathrm{Bad}: a_{1}(x)=2 \rightarrow d(x)=\mathrm{Bad}$ or $a_{2}(x)=5 \rightarrow d(x)=\mathrm{Bad}$ or $a_{3}(x)=3 \rightarrow$ $d(x)=\mathrm{Bad} / /$ supported by $x_{6} \in{\overline{\sum_{i=1}^{3} a_{i}}}^{P}(\mathrm{Bad})$

7. $\bigvee_{i=1}^{3}\left[x_{7}\right]_{a_{i}} \rightarrow \mathrm{Bad}: a_{1}(x)=3 \rightarrow d(x)=\mathrm{Bad}$ or $a_{2}(x)=1 \rightarrow d(x)=\mathrm{Bad}$ or $a_{3}(x)=2 \rightarrow$ $d(x)=\mathrm{Bad} / /$ supported by $x_{7} \in{\overline{\sum_{i=1}^{3} a_{i}}}^{P}(\mathrm{Bad})$

8. $\bigvee_{i=1}^{3}\left[x_{8}\right]_{a_{i}} \rightarrow \mathrm{Bad}: a_{1}(x)=2 \rightarrow d(x)=\mathrm{Bad}$ or $a_{2}(x)=1 \rightarrow d(x)=$ Bad or $a_{3}(x)=2 \rightarrow$ $d(x)=\mathrm{Bad} / /$ supported by $x_{8} \in{\overline{\sum_{i=1}^{3} a_{i}}}^{P}(\mathrm{Bad})$

9. $\bigvee_{i=1}^{3}\left[x_{1}\right]_{a_{i}} \rightarrow$ Medium: $a_{1}(x)=2 \rightarrow d(x)=$ Medium or $a_{2}(x)=3 \rightarrow d(x)=$ Medium or $a_{3}(x)=2 \rightarrow d(x)=$ Meidum // supported by $x_{1} \in{\overline{\sum_{i=1}^{3} a_{i}}}^{P}$ (Medium) 
10. $\bigvee_{i=1}^{3}\left[x_{2}\right]_{a_{i}} \rightarrow$ Medium: $a_{1}(x)=5 \rightarrow d(x)=$ Medium or $a_{2}(x)=1 \rightarrow d(x)=$ Medium or $a_{3}(x)=3 \rightarrow P(x)=$ Meidum // supported by $x_{2} \in{\overline{\sum_{i=1}^{3} a_{i}}}^{P}$ (Medium)

11. $\bigvee_{i=1}^{3}\left[x_{3}\right]_{a_{i}} \rightarrow$ Medium: $a_{1}(x)=5 \rightarrow d(x)=$ Medium or $a_{2}(x)=2 \rightarrow d(x)=$ Medium or $a_{3}(x)=4 \rightarrow d(x)=$ Meidum // supported by $x_{3} \in{\overline{\sum_{i=1}^{3} a_{i}}}^{P}$ (Medium)

12. $\bigvee_{i=1}^{3}\left[x_{4}\right]_{a_{i}} \rightarrow$ Medium: $a_{1}(x)=3 \rightarrow d(x)=$ Medium or $a_{2}(x)=5 \rightarrow d(x)=$ Medium or $a_{3}(x)=3 \rightarrow d(x)=$ Meidum // supported by $x_{4} \in{\overline{\sum_{i=1}^{3} a_{i}}}^{P}$ (Medium)

13. $\bigvee_{i=1}^{3}\left[x_{6}\right]_{a_{i}} \rightarrow$ Medium: $a_{1}(x)=2 \rightarrow d(x)=$ Medium or $a_{2}(x)=5 \rightarrow d(x)=$ Medium or $a_{3}(x)=3 \rightarrow d(x)=$ Medium // supported by $x_{6} \in{\overline{\sum_{i=1}^{3} a_{i}}}^{P}$ (Medium)

14. $\bigvee_{i=1}^{3}\left[x_{7}\right]_{a_{i}} \rightarrow$ Medium: $a_{1}(x)=3 \rightarrow d(x)=$ Medium or $a_{2}(x)=1 \rightarrow d(x)=$ Medium or $a_{3}(x)=2 \rightarrow P(x)=$ Meidum // supported by $x_{7} \in{\overline{\sum_{i=1}^{3} a_{i}}}^{P}$ (Medium)

15. $\bigvee_{i=1}^{3}\left[x_{8}\right]_{a_{i}} \rightarrow$ Medium: $a_{1}(x)=2 \rightarrow d(x)=$ Medium or $a_{2}(x)=1 \rightarrow d(x)=$ Medium or $a_{3}(x)=2 \rightarrow d(x)=$ Meidum // supported by $x_{8} \in{\overline{\sum_{i=1}^{3} a_{i}}}^{P}$ (Medium)

16. $\bigvee_{i=1}^{3}\left[x_{2}\right]_{a_{i}} \rightarrow$ Good: $a_{1}(x)=5 \rightarrow d(x)=$ Good or $a_{2}(x)=1 \rightarrow d(x)=$ Good or $a_{3}(x)=$ $3 \rightarrow d(x)=$ Good // supported by $x_{2} \in$ ${\overline{\sum_{i=1}^{3} a_{i}}}^{P}(\mathrm{Good})$

17. $\bigvee_{i=1}^{3}\left[x_{4}\right]_{a_{i}} \rightarrow$ Good: $a_{1}(x)=3 \rightarrow d(x)=$ Good or $a_{2}(x)=5 \rightarrow d(x)=$ Good or $a_{3}(x)=$ $3 \rightarrow d(x)=$ Good // supported by $x_{4} \in$ ${\overline{\sum_{i=1}^{3} a_{i}}}^{P}(\mathrm{Good})$

18. $\bigvee_{i=1}^{3}\left[x_{6}\right]_{a_{i}} \rightarrow$ Good: $a_{1}(x)=2 \rightarrow d(x)=$ Good or $a_{2}(x)=5 \rightarrow d(x)=$ Good or $a_{3}(x)=$ $3 \rightarrow d(x)=$ Good // supported by $x_{6} \in$ ${\overline{\sum_{i=1}^{3} a_{i}}}^{P}(\mathrm{Good})$
19. $\bigvee_{i=1}^{3}\left[x_{7}\right]_{a_{i}} \rightarrow$ Good: $a_{1}(x)=3 \rightarrow d(x)=$ Good or $a_{2}(x)=1 \rightarrow d(x)=$ Good or $a_{3}(x)=$ $2 \rightarrow d(x)=$ Good // supported by $x_{7} \in$ ${\overline{\sum_{i=1}^{3} a_{i}}}^{P}(\mathrm{Good})$

“AND” MGRS rules:

1. $\bigwedge_{i=1}^{3}\left[x_{1}\right]_{a_{i}} \rightarrow$ Bad: $a_{1}(x)=2 \rightarrow d(x)=\mathrm{Bad}$ and $a_{2}(x)=3 \rightarrow d(x)=$ Bad and $a_{3}(x)=$ $2 \rightarrow d(x)=\mathrm{Bad} / /$ supported by $x_{1} \in$ ${\overline{\sum_{i=1}^{3} a_{i}}}^{O}(\mathrm{Bad})$

2. $\bigwedge_{i=1}^{3}\left[x_{3}\right]_{a_{i}} \rightarrow$ Bad: $a_{1}(x)=5 \rightarrow d(x)=\mathrm{Bad}$ and $a_{2}(x)=2 \rightarrow d(x)=$ Bad and $a_{3}(x)=$ $4 \rightarrow d(x)=\mathrm{Bad} / /$ supported by $x_{3} \in$ ${\overline{\sum_{i=1}^{3} a_{i}}}^{O}(\mathrm{Bad})$

3. $\bigwedge_{i=1}^{3}\left[x_{5}\right]_{a_{i}} \rightarrow$ Bad: $a_{1}(x)=1 \rightarrow d(x)=\mathrm{Bad}$ and $a_{2}(x)=3 \rightarrow d(x)=\mathrm{Bad}$ and $a_{3}(x)=$ $4 \rightarrow d(x)=\mathrm{Bad} / /$ supported by $x_{5} \in$ $\underline{\sum_{i=1}^{3} a_{i}^{P}}(\mathrm{Bad}),{\overline{\sum_{i=1}^{3} a_{i}}}^{O}(\mathrm{Bad})$

4. $\bigwedge_{i=1}^{3}\left[x_{7}\right]_{a_{i}} \rightarrow \mathrm{Bad}: a_{1}(x)=3 \rightarrow d(x)=\mathrm{Bad}$ and $a_{2}(x)=1 \rightarrow d(x)=\mathrm{Bad}$ and $a_{3}(x)=$ $2 \rightarrow d(x)=\mathrm{Bad} / /$ supported by $x_{7} \in$ ${\overline{\sum_{i=1}^{3} a_{i}}}^{O}(\mathrm{Bad})$

5. $\bigwedge_{i=1}^{3}\left[x_{8}\right]_{a_{i}} \rightarrow \mathrm{Bad}: a_{1}(x)=2 \rightarrow d(x)=\mathrm{Bad}$ and $a_{2}(x)=1 \rightarrow d(x)=$ Bad and $a_{3}(x)=$ $2 \rightarrow d(x)=$ Bad // supported by $x_{8} \in$ ${\overline{\sum_{i=1}^{3} a_{i}}}^{O}(\mathrm{Bad})$

6. $\bigwedge_{i=1}^{3}\left[x_{2}\right]_{a_{i}} \rightarrow$ Medium: $a_{1}(x)=5 \rightarrow d(x)=$ Medium and $a_{2}(x)=1 \rightarrow d(x)=$ Medium and $a_{3}(x)=3 \rightarrow d(x)=$ Medium // supported by $x_{2} \in{\overline{\sum_{i=1}^{3} a_{i}}}^{O}$ (Medium)

7. $\bigwedge_{i=1}^{3}\left[x_{6}\right]_{a_{i}} \rightarrow$ Medium: $a_{1}(x)=2 \rightarrow d(x)=$ Medium and $a_{2}(x)=5 \rightarrow d(x)=$ Medium and $a_{3}(x)=3 \rightarrow d(x)=$ Medium // supported by $x_{6} \in{\overline{\sum_{i=1}^{3} a_{i}}}^{O}$ (Medium)

8. $\bigwedge_{i=1}^{3}\left[x_{8}\right]_{a_{i}} \rightarrow$ Medium: $a_{1}(x)=2 \rightarrow d(x)=$ Medium and $a_{2}(x)=1 \rightarrow d(x)=$ Medium and $a_{3}(x)=2 \rightarrow d(x)=$ Medium // supported by $x_{8} \in{\overline{\sum_{i=1}^{3} a_{i}}}^{O}$ (Medium) 
9. $\bigwedge_{i=1}^{3}\left[x_{4}\right]_{a_{i}} \rightarrow$ Good: $\quad a_{1}(x)=3 \rightarrow d(x)=$ Good and $a_{2}(x)=5 \rightarrow d(x)=$ Good and $a_{3}(x)=3 \rightarrow d(x)=$ Good // supported by $x_{4} \in{\overline{\sum_{i=1}^{3} a_{i}}}^{O}$ (Good)

Table 2. Three local factors for MGRS rules in Table 1 .

\begin{tabular}{lccc}
\hline MGRS rules & Supports & Certainty & Coverage \\
\hline$\bigvee_{i=1}^{3}\left[x_{1}\right] a_{i} \rightarrow$ Bad & 0.25 & 1 & 0.5 \\
$\bigvee_{i=1}^{3}\left[x_{2}\right] a_{i} \rightarrow$ Bad & 0.125 & 0.5 & 0.25 \\
$\bigvee_{i=1}^{3}\left[x_{3}\right] a_{i} \rightarrow$ Bad & 0.25 & 1 & 0.5 \\
$\bigvee_{i=1}^{3}\left[x_{4}\right] a_{i} \rightarrow$ Bad & 0.125 & 0.5 & 0.25 \\
$\bigvee_{i=1}^{3}\left[x_{5}\right] a_{i} \rightarrow$ Bad & 0.25 & 1 & 0.5 \\
$\bigvee_{i=1}^{3}\left[x_{6}\right] a_{i} \rightarrow$ Bad & 0.125 & 0.33 & 0.25 \\
$\bigvee_{i=1}^{3}\left[x_{7}\right] a_{i} \rightarrow$ Bad & 0.25 & 0.67 & 0.5 \\
$\bigvee_{i=1}^{3}\left[x_{8}\right] a_{i} \rightarrow$ Bad & 0.25 & 0.67 & 0.5 \\
$\bigvee_{i=1}^{3}\left[x_{1}\right] a_{i} \rightarrow$ Medium & 0.25 & 0.67 & 0.67 \\
$\bigvee_{i=1}^{3}\left[x_{2}\right] a_{i} \rightarrow$ Medium & 0.25 & 0.67 & 0.67 \\
$\bigvee_{i=1}^{3}\left[x_{3}\right] a_{i} \rightarrow$ Medium & 0.125 & 0.5 & 0.33 \\
$\bigvee_{i=1}^{3}\left[x_{4}\right] a_{i} \rightarrow$ Medium & 0.25 & 0.67 & 0.67 \\
$\bigvee_{i=1}^{3}\left[x_{6}\right] a_{i} \rightarrow$ Medium & 0.25 & 0.67 & 0.67 \\
$\bigvee_{i=1}^{3}\left[x_{7}\right] a_{i} \rightarrow$ Medium & 0.25 & 0.67 & 0.67 \\
$\bigvee_{i=1}^{3}\left[x_{8}\right] a_{i} \rightarrow$ Medium & 0.25 & 0.67 & 0.67 \\
$\bigvee_{i=1}^{3}\left[x_{2}\right] a_{i} \rightarrow$ Good & 0.125 & 0.33 & 1 \\
$\bigvee_{i=1}^{3}\left[x_{4}\right] a_{i} \rightarrow$ Good & 0.125 & 0.5 & 1 \\
$\bigvee_{i=1}^{3}\left[x_{6}\right] a_{i} \rightarrow$ Good & 0.125 & 0.5 & 1 \\
$\bigvee_{i=1}^{3}\left[x_{7}\right] a_{i} \rightarrow$ Good & 0.125 & 0.5 & 1 \\
$\bigwedge_{i=1}^{3}\left[x_{1}\right] a_{a_{i}} \rightarrow$ Bad & 0.125 & 0.33 & 0.25 \\
$\bigwedge_{i=1}^{3}\left[x_{3}\right] a_{i} \rightarrow$ Bad & 0.125 & 0.5 & 0.25 \\
$\bigwedge_{i=1}^{3}\left[x_{5}\right] a_{i} \rightarrow$ Bad & 0.125 & 1 & 0.25 \\
$\bigwedge_{i=1}^{3}\left[x_{7}\right] a_{i} \rightarrow$ Bad & 0.125 & 0.33 & 0.25 \\
$\bigwedge_{i=1}^{3}\left[x_{8}\right] a_{i} \rightarrow$ Bad & 0.125 & 0.33 & 0.25 \\
$\bigwedge_{i=1}^{3}\left[x_{2}\right] a_{a_{i}} \rightarrow$ Medium & 0.125 & 0.5 & 0.33 \\
$\bigwedge_{i=1}^{3}\left[x_{6}\right] a_{i} \rightarrow$ Medium & 0.125 & 0.5 & 0.33 \\
$\bigwedge_{i=1}^{3}\left[x_{8}\right] a_{i} \rightarrow$ Medium & 0.125 & 0.33 & 0.33 \\
$\bigwedge_{i=1}^{3}\left[x_{4}\right] a_{i} \rightarrow$ Good & 0.125 & 0.33 & 1 \\
\hline & & &
\end{tabular}

Table 2 shows the support, certainty and coverage factors of the MGRS rules in Table 1.

\subsection{Global measuring of MGRS rules}

Obviously, the three factors defined in Definition 4 can only be used to measure a single MGRS rules. Through the example shown in the last subsection, we can see that given a decision system, a family of the MGRS rules may be unraveled. Therefore, how to measure these MGRS rules from a global view has become a necessity. This is what will be discussed in the following.

Definition 5. Let $I$ be a decision system, in which $A_{1}, A_{2}, \cdots, A_{m} \subseteq A T$ and $U / \operatorname{IND}(\{d\})=$ $\left\{X_{1}, X_{2}, \cdots, X_{k}\right\}$, then
1. the global support factor of "OR" MGRS rules in $I$ is

$\alpha^{\vee}=\frac{1}{|U|} \sum_{x \in U} \frac{1}{k} \sum_{X_{j} \in U / I N D(\{d\})} \operatorname{Supp}\left(\bigvee_{i=1}^{m}[x]_{A_{i}} \rightarrow X_{j}\right)$,

2. the global support factor of "AND" MGRS rules in $I$ is

$$
\alpha^{\wedge}=\frac{1}{|U|} \sum_{x \in U} \frac{1}{k} \sum_{X_{j} \in U / I N D(\{d\})} \operatorname{Supp}\left(\bigwedge_{i=1}^{m}[x]_{A_{i}} \rightarrow X_{j}\right),
$$

3. the global certainty factor of "OR" MGRS rules in $I$ is

$$
\beta^{\vee}=\frac{1}{|U|} \sum_{x \in U} \frac{1}{k} \sum_{X_{j} \in U / I N D(\{d\})} \operatorname{Cer}\left(\bigvee_{i=1}^{m}[x]_{A_{i}} \rightarrow X_{j}\right),
$$

4. the global certainty factor of "AND" MGRS rules in $I$ is

$$
\beta^{\wedge}=\frac{1}{|U|} \sum_{x \in U} \frac{1}{k} \sum_{X_{j} \in U / I N D(\{d\})} \operatorname{Cer}\left(\bigwedge_{i=1}^{m}[x]_{A_{i}} \rightarrow X_{j}\right),
$$

5. the global coverage factor of "OR" MGRS rules in $I$ is

$$
\gamma^{\vee}=\frac{1}{|U|} \sum_{x \in U} \frac{1}{k} \sum_{X_{j} \in U / I N D(\{d\})} \operatorname{Cov}\left(\bigvee_{i=1}^{m}[x]_{A_{i}} \rightarrow X_{j}\right),
$$

6. the global coverage factor of "AND" MGRS rules in $I$ is

$$
\gamma^{\wedge}=\frac{1}{|U|} \sum_{x \in U} \frac{1}{k} \sum_{X_{j} \in U / I N D(\{d\})} \operatorname{Cov}\left(\bigwedge_{i=1}^{m}[x]_{A_{i}} \rightarrow X_{j}\right) .
$$

Theorem 8. Let I be a decision system, in which $A_{1}, A_{2}, \cdots, A_{m} \subseteq A T$ and $U / I N D(\{d\})=$ $\left\{X_{1}, X_{2}, \cdots, X_{k}\right\}$, then

1. $\alpha^{\vee} \in\left[\frac{1}{k|U|}, 1\right]$;

2. $\alpha^{\wedge} \in\left[\frac{1}{k|U|}, 1\right]$. 
Proof. We only prove 1, the proof of 2 is similar to the proof of 1 .

If $\forall x \in U$ and $\forall X_{j} \in U / \operatorname{IND}(\{d\})$, $\operatorname{Supp}\left(\bigvee_{i=1}^{m}[x]_{A_{i}} \rightarrow X_{j}\right)$ achieves its minimal value, then $\alpha^{\vee}$ will achieve its minimal value. By Definition 4, we know that $\operatorname{Supp}\left(\bigvee_{i=1}^{m}[x]_{A_{i}} \rightarrow X_{j}\right) \in[0,1]$, i.e. the minimal value of $\operatorname{Supp}\left(\bigvee_{i=1}^{m}[x]_{A_{i}} \rightarrow X_{j}\right)$ is 0 . However, it should be noticed that since the decision attribute determine a partition on the universe of discourse, then $\forall x \in U$, there must be $X_{l} \in U / I N D(\{d\})$ such that $x \in X_{l}$. In such case, the minimal value is $\operatorname{Supp}\left(\bigvee_{i=1}^{m}[x]_{A_{i}} \rightarrow X_{l}\right)=\frac{1}{|U|}$. From discussions above, if $\forall j \neq l, \operatorname{Supp}\left(\bigvee_{i=1}^{m}[x]_{A_{i}} \rightarrow X_{j}\right)$ achieves its minimal value 0 , then $\alpha^{\vee}$ will achieves its minimal value $\frac{1}{k|U|}$.

On the other hand, if $\forall x \in U$ and $\forall X_{j} \in$ $U / \operatorname{IND}(\{d\}), \operatorname{Supp}\left(\bigvee_{i=1}^{m}[x]_{A_{i}} \rightarrow X_{j}\right)$ achieves its maximal value, then $\alpha^{\vee}$ will achieve its maximal value. By Definition 4, we know that $\operatorname{Supp}\left(\bigvee_{i=1}^{m}[x]_{A_{i}} \rightarrow X_{j}\right) \in[0,1]$, i.e. the maximal value of $\operatorname{Supp}\left(\bigvee_{i=1}^{m}[x]_{A_{i}} \rightarrow X_{j}\right)$ is 1. Therefore, the maximal value of $\alpha^{\vee}$ is 1 .

Theorem 8 shows that the global support factors of "OR" and "AND" MGRS rules are between the interval $\left[\frac{1}{k|U|}, 1\right]$.

Theorem 9. Let I be a decision system, in which $A_{1}, A_{2}, \cdots, A_{m} \subseteq A T$ and $U / I N D(\{d\})=$ $\left\{X_{1}, X_{2}, \cdots, X_{k}\right\}$,

1. if $\forall i \in\{1,2, \cdots, m\}, \quad U / \operatorname{IND}\left(A_{i}\right)=$ $\left\{\left\{x_{1}\right\},\left\{x_{2}\right\}, \cdots,\left\{x_{|U|}\right\}\right\}$, then $\alpha^{\vee}$ achieves its minimal value $\frac{1}{k|U|}$;

2. if $\exists i \in\{1,2, \cdots, m\}, U / \operatorname{IND}\left(A_{i}\right)=U$ and $U / I N D(\{d\})=U$, then $\alpha^{\vee}$ achieves its maximal value 1 ;

3. if $\exists i \in\{1,2, \cdots, m\}, \quad U / \operatorname{IND}\left(A_{i}\right)=$ $\left\{\left\{x_{1}\right\},\left\{x_{2}\right\}, \cdots,\left\{x_{|U|}\right\}\right\}$, then $\alpha^{\wedge}$ achieves its minimal value $\frac{1}{k|U|}$;

4. if $\forall i \in\{1,2, \cdots, m\}, U / I N D\left(A_{i}\right)=U$ and $U / I N D(\{d\})=U$, then $\alpha^{\wedge}$ achieves its maximal value 1 .

Proof. We only prove 1, others can be proved analogously.
By condition, $\forall i \in\{1,2, \cdots, m\}, U / \operatorname{IND}\left(A_{i}\right)=$ $\left\{\left\{x_{1}\right\},\left\{x_{2}\right\}, \cdots,\left\{x_{|U|}\right\}\right\}$, then we know that $[x]_{A_{i}}=$ $\{x\}$ for each $x$ and $i \in\{1,2, \cdots, m\}$, thus, $\forall X_{j} \in$ $U / I N D(\{d\})$, if $x \in X_{j}$, then $\operatorname{Supp}\left(\bigvee_{i=1}^{m}[x]_{A_{i}} \rightarrow\right.$ $\left.X_{j}\right)=\frac{1}{|U|}$; if $x \notin X_{j}$, then $\operatorname{Supp}\left(\bigvee_{i=1}^{m}[x]_{A_{i}} \rightarrow X_{j}\right)=0$; it follows that $\frac{1}{k} \sum_{X_{j} \in U / I N D(\{d\})} \operatorname{Supp}\left(\bigvee_{i=1}^{m}[x]_{A_{i}} \rightarrow\right.$ $\left.X_{j}\right)=\frac{1}{k|U|}$ for each $x \in U$ and then $\alpha^{\vee}$ achieves its minimal value $\frac{1}{k|U|}$.

Theorem 9 provides the necessary conditions for the global support factors of "OR" and "AND" MGRS rules to achieve their maximal and minimal values.

Theorem 10. Let I be a decision system, in which $A_{1}, A_{2}, \cdots, A_{m} \subseteq A T$ and $U / I N D(\{d\})=$ $\left\{X_{1}, X_{2}, \cdots, X_{k}\right\}$, then

1. $\beta^{\vee} \in\left[\frac{1}{|U|}, 1\right]$;

2. $\beta^{\wedge} \in\left[\frac{1}{|U|}, 1\right]$.

Proof. We only prove 1 , the proof of 2 is similar to the proof of 1 .

If $\forall x \in U$ and $\forall X_{j} \in U / \operatorname{IND}(\{d\})$, $\operatorname{Cer}\left(\bigvee_{i=1}^{m}[x]_{A_{i}} \rightarrow X_{j}\right)$ achieves its minimal value, then $\beta^{\vee}$ will achieve its minimal value. By Definition 4, we know that $\operatorname{Cer}\left(\bigvee_{i=1}^{m}[x]_{A_{i}} \rightarrow X_{j}\right) \in[0,1]$, i.e. the minimal value of $\operatorname{Cer}\left(\bigvee_{i=1}^{m}[x]_{A_{i}} \rightarrow X_{j}\right)$ is 0 . However, it should be noticed that since the decision attribute determine a partition on the universe of discourse, then $\forall x \in U$, there must be $X_{l} \in U / I N D(\{d\})$ such that $x \in X_{l}$. In such case, the minimal value is $\operatorname{Cer}\left(\bigvee_{i=1}^{m}[x]_{A_{i}} \rightarrow X_{l}\right)=\frac{1}{|U|}$. Moreover, since $\operatorname{Cer}\left(\bigvee_{i=1}^{m}[x]_{A_{i}} \rightarrow X_{l}\right)=\frac{1}{|U|}$, then by Definition 4, we know that $\forall i \in\{1,2, \cdots, m\}$, we have $[x]_{A_{i}}=|U|$, thus, $\forall j \neq l$, the minimal value of $\operatorname{Cer}\left(\bigvee_{i=1}^{m}[x]_{A_{i}} \rightarrow X_{j}\right)$ is also $\frac{1}{\mid U}$, then $\beta^{\vee}$ will achieves its minimal value $\frac{1}{|U|}$.

On the other hand, if $\forall x \in U$ and $\forall X_{j} \in$ $U / I N D(\{d\}), \operatorname{Cer}\left(\bigvee_{i=1}^{m}[x]_{A_{i}} \rightarrow X_{j}\right)$ achieves it$\mathrm{s}$ maximal value, then $\beta^{\vee}$ will achieve its maximal value. By Definition 4, we know that $\operatorname{Cer}\left(\bigvee_{i=1}^{m}[x]_{A_{i}} \rightarrow X_{j}\right) \in[0,1]$, i.e. the maximal value of $\operatorname{Cer}\left(\bigvee_{i=1}^{m}[x]_{A_{i}} \rightarrow X_{j}\right)$ is 1 . Therefore, the maximal value of $\beta^{\vee}$ is 1 . 
Theorem 10 shows that the global certainty factors of "OR" and "AND" MGRS rules are between the interval $\left[\frac{1}{|U|}, 1\right]$.

Theorem 11. Let I be a decision system, in which $A_{1}, A_{2}, \cdots, A_{m} \subseteq A T$ and $U / \operatorname{IND}(\{d\})=$ $\left\{X_{1}, X_{2}, \cdots, X_{k}\right\}$,

1. if $\forall i \in\{1,2, \cdots, m\}, U / I N D\left(A_{i}\right)=U$ and $U / \operatorname{IND}(\{d\})=\left\{\left\{x_{1}\right\},\left\{x_{2}\right\}, \cdots,\left\{x_{|U|}\right\}\right\}$, then $\beta^{\vee}$ achieves its minimal value $\frac{1}{|U|}$;

2. if $\exists i \in\{1,2, \cdots, m\}, \quad U / \operatorname{IND}\left(A_{i}\right)=$ $\left\{\left\{x_{1}\right\},\left\{x_{2}\right\}, \cdots,\left\{x_{|U|}\right\}\right\}$ and $U / \operatorname{IND}(\{d\})=$ $U$, then $\beta^{\vee}$ achieves its maximal value 1 ;

3. if $\exists i \in\{1,2, \cdots, m\}, U / I N D\left(A_{i}\right)=U$ and $U / \operatorname{IND}(\{d\})=\left\{\left\{x_{1}\right\},\left\{x_{2}\right\}, \cdots,\left\{x_{|U|}\right\}\right.$, then $\beta^{\wedge}$ achieves its minimal value $\frac{1}{|U|}$;

4. if $\forall i \in\{1,2, \cdots, m\}, \quad U / \operatorname{IND}\left(A_{i}\right)=$ $\left\{\left\{x_{1}\right\},\left\{x_{2}\right\}, \cdots,\left\{x_{|U|}\right\}\right\}$ and $U / \operatorname{IND}(\{d\})=$ $U$, then $\beta^{\wedge}$ achieves its maximal value 1 .

Proof. We only prove 1 , others can be proved analogously.

By condition, $\forall i \in\{1,2, \cdots, m\}, U / I N D\left(A_{i}\right)=$ $U$, then we know that $[x]_{A_{i}}=U$ for each $x$ and $\forall i \in\{1,2, \cdots, m\}$. Moreover, since $U / \operatorname{IND}(\{d\})=$ $\left\{\left\{x_{1}\right\},\left\{x_{2}\right\}, \cdots,\left\{x_{|U|}\right\}\right\}$, then $\forall X_{j} \in U / I N D(\{d\})$, $X_{j}$ is the subset of $U$ with single object. Therefore, $\operatorname{Cer}\left(\bigvee_{i=1}^{m}[x]_{A_{i}} \rightarrow X_{j}\right)=\frac{1}{|U|}$; it follows that $\frac{1}{k} \sum_{X_{j} \in U / I N D(\{d\})} \operatorname{Cer}\left(\bigvee_{i=1}^{m}[x]_{A_{i}} \rightarrow X_{j}\right)=\frac{1}{|U|}$ for each $x \in U$ and then $\beta^{\vee}$ achieves its minimal value $\frac{1}{|U|}$. $\square$

Theorem 11 provides the necessary conditions for the global certainty factors of "OR" and "AND" MGRS rules to achieve its maximal and minimal values.

Theorem 12. Let I be a decision system, in which $A_{1}, A_{2}, \cdots, A_{m} \subseteq A T$ and $U / I N D(\{d\})=$ $\left\{X_{1}, X_{2}, \cdots, X_{k}\right\}$, then

1. $\gamma^{\vee} \in\left[\frac{1}{|U|}, 1\right]$;

2. $\gamma^{\wedge} \in\left[\frac{1}{|U|}, 1\right]$.
Proof. We only prove 1 , the proof of 2 is similar to the proof of 1 .

If $\forall x \in U$ and $\forall X_{j} \in U / \operatorname{IND}(\{d\})$, $\operatorname{Cov}\left(\bigvee_{i=1}^{m}[x]_{A_{i}} \rightarrow X_{j}\right)$ achieves its minimal value, then $\gamma^{\vee}$ will achieve its minimal value. By Definition 4, we know that $\operatorname{Cov}\left(\bigvee_{i=1}^{m}[x]_{A_{i}} \rightarrow X_{j}\right) \in[0,1]$, i.e. the minimal value of $\operatorname{Cov}\left(\bigvee_{i=1}^{m}[x]_{A_{i}} \rightarrow X_{j}\right)$ is 0 . However, it should be noticed that since the decision attribute determine a partition on the universe of discourse, then $\forall x \in U$, there must be $X_{l} \in$ $U / I N D(\{d\})$ such that $x \in X_{l}$. In such case, the minimal value is $\operatorname{Cov}\left(\bigvee_{i=1}^{m}[x]_{A_{i}} \rightarrow X_{l}\right)=\frac{1}{|U|}$. Moreover, since $\operatorname{Cov}\left(\bigvee_{i=1}^{m}[x]_{A_{i}} \rightarrow X_{l}\right)=\frac{1}{|U|}$, then by Definition 4, we know that $U / \operatorname{IND}(\{d\})=U=\left\{X_{l}\right\}$, thus, $\gamma^{\vee}=\frac{1}{|U|} \sum_{x \in U} \frac{1}{k} \sum_{X_{j} \in U / I N D(\{d\})} \operatorname{Cov}\left(\bigvee_{i=1}^{m}[x]_{A_{i}} \rightarrow\right.$ $\left.X_{j}\right)=\frac{1}{|U|} \sum_{x \in U} \operatorname{Cov}\left(\bigvee_{i=1}^{m}[x]_{A_{i}} \rightarrow U\right)$. If $\forall x \in U$, $\operatorname{Cov}\left(\bigvee_{i=1}^{m}[x]_{A_{i}} \rightarrow U\right)$ achieve its minimal value $\frac{1}{|U|}$, then $\gamma^{\vee}$ will achieve its minimal value $\frac{1}{|U|}$.

On the other hand, if $\forall x \in U$ and $\forall X_{j} \in$ $U / I N D(\{d\}), \operatorname{Cov}\left(\bigvee_{i=1}^{m}[x]_{A_{i}} \rightarrow X_{j}\right)$ achieves it$\mathrm{s}$ maximal value, then $\gamma^{\vee}$ will achieve its maximal value. By Definition 4, we know that $\operatorname{Cov}\left(\bigvee_{i=1}^{m}[x]_{A_{i}} \rightarrow X_{j}\right) \in[0,1]$, i.e. the maximal value of $\operatorname{Cov}\left(\bigvee_{i=1}^{m}[x]_{A_{i}} \rightarrow X_{j}\right)$ is 1 . Therefore, the maximal value of $\gamma^{\vee}$ is 1 .

Theorem 12 shows that the global coverage factors of "OR" and "AND" MGRS rules are between the interval $\left[\frac{1}{|U|}, 1\right]$.

Theorem 13. Let I be a decision system, in which $A_{1}, A_{2}, \cdots, A_{m} \subseteq A T$ and $U / I N D(\{d\})=$ $\left\{X_{1}, X_{2}, \cdots, X_{k}\right\}$,

1. if $\forall i \in\{1,2, \cdots, m\}, \quad U / \operatorname{IND}\left(A_{i}\right)=$ $\left\{\left\{x_{1}\right\},\left\{x_{2}\right\}, \cdots,\left\{x_{|U|}\right\}\right\}$ and $U / \operatorname{IND}(\{d\})=$ $U$, then $\gamma^{\vee}$ achieves its minimal value $\frac{1}{|U|}$;

2. if $\exists i \in\{1,2, \cdots, m\}, U / \operatorname{IND}\left(A_{i}\right)=U$ and $U / I N D(\{d\})=U$, then $\gamma^{\vee}$ achieves its maximal value 1 ;

3. if $\exists i \in\{1,2, \cdots, m\}, \quad U / \operatorname{IND}\left(A_{i}\right)=$ $\left\{\left\{x_{1}\right\},\left\{x_{2}\right\}, \cdots,\left\{x_{|U|}\right\}\right\}$ and $U / \operatorname{IND}(\{d\})=$ $U$, then $\gamma^{\wedge}$ achieves its minimal value $\frac{1}{|U|}$;

4. if $\forall i \in\{1,2, \cdots, m\}, U / \operatorname{IND}\left(A_{i}\right)=U$ and 
$U / \operatorname{IND}(\{d\})=U$, then $\gamma^{\wedge}$ achieves its maximal value 1 .

Proof. We only prove 1 , others can be proved analogously.

By condition, $\forall i \in\{1,2, \cdots, m\}, U / \operatorname{IND}\left(A_{i}\right)=$ $\left\{\left\{x_{1}\right\},\left\{x_{2}\right\}, \cdots,\left\{x_{|U|}\right\}\right\}$, then we know that $[x]_{A_{i}}=$ $\{x\}$ for each $x$ and $i \in\{1,2, \cdots, m\}$. Moreover, since $U / I N D(\{d\})=U$, then $\forall X_{k} \in U / I N D(\{d\}), X_{k}=$ $U$. Therefore, $\operatorname{Cov}\left(\bigvee_{i=1}^{m}[x]_{A_{i}} \rightarrow U\right)=\frac{1}{|U|}$; it follows that $\frac{1}{k} \sum_{X_{k} \in U / I N D(\{d\})} \operatorname{Cov}\left(\bigvee_{i=1}^{m}[x]_{A_{i}} \rightarrow X_{k}\right)=\frac{1}{|U|}$ for each $x \in U$ and then $\gamma^{\vee}$ achieves its minimal value $\frac{1}{|U|}$.

Theorem 13 provides the necessary conditions for the global coverage factors of "OR" and "AND" MGRS rules to achieve its maximal and minimal values.

Example 1. Take for instance Table 1, compute the global support factors, global certainty factors and global coverage factors for the "OR" and "AND" MGRS rules in Table 1.

Obviously, if different sets of attributes are employed, then different "OR" and "AND" MGRS rules can be derived, it follows that the different factors may be obtained.

For example, if the used sets of attributes are $A_{1}=\left\{a_{1}\right\}, A_{2}=\left\{a_{2}\right\}, A_{3}=\left\{a_{3}\right\}$, then by Definition 5 , following the computing results of Table 2 , we have

$$
\begin{aligned}
\alpha^{\vee} & =\frac{1}{|U|} \sum_{x \in U} \frac{1}{k} \sum_{X_{j} \in U / I N D(\{d\})} \operatorname{Supp}\left(\bigvee_{i=1}^{m}[x]_{A_{i}} \rightarrow X_{j}\right) \\
& =\frac{1}{8} * \frac{1}{3} * 3.75 \\
& \approx 0.16 .
\end{aligned}
$$

Similarity, it is not difficult to obtain that $\alpha^{\wedge}=$ $0.05, \beta^{\vee}=0.5, \beta^{\wedge}=0.17, \gamma^{\vee}=0.49, \gamma^{\wedge}=0.14$.

If the used sets of attributes are $A_{1}=\left\{a_{1}, a_{2}, a_{3}\right\}$, then $\alpha^{\vee}=0.04, \alpha^{\wedge}=0.04, \beta^{\vee}=0.44, \beta^{\wedge}=0.44$, $\gamma^{\vee}=0.125, \gamma^{\wedge}=0.125$.

If the used sets of attributes are $A_{1}=$ $\left\{a_{1}, a_{2}\right\}, A_{2}=\left\{a_{3}\right\}$, then $\alpha^{\vee}=0.11, \alpha^{\wedge}=0.04$, $\beta^{\vee}=0.44, \beta^{\wedge}=0.33, \gamma^{\vee}=0.35, \gamma^{\wedge}=0.125$.
If the used sets of attributes are $A_{1}=\left\{a_{1}\right\}, A_{2}=$ $\left\{a_{2}, a_{3}\right\}$, then $\alpha^{\vee}=0.11, \alpha^{\wedge}=0.05, \beta^{\vee}=0.47$, $\beta^{\wedge}=0.19, \gamma^{\vee}=0.35, \gamma^{\wedge}=0.14$.

If the used sets of attributes are $A_{1}=$ $\left\{a_{1}, a_{3}\right\}, A_{2}=\left\{a_{2}\right\}$, then $\alpha^{\vee}=0.10, \alpha^{\wedge}=0.05$, $\beta^{\vee}=0.44, \beta^{\wedge}=0.22, \gamma^{\vee}=0.29, \gamma^{\wedge}=0.14$.

Example 2. Venture capital has become an increasingly important source of financing for new companies, particularly when such companies are operating on the frontier of emerging technologies and markets. It plays an essential role in the entrepreneurial process. In the following, we will use a venture investment issue which was showed in Ref. ${ }^{16}$, to illustrate the three global measurements.

Let us consider a real investment issue of a venture investment company (Here we conceal the company's name and the details of investmen$\mathrm{t}$ projects). There are fifty investment projects $x_{i}$ $(i=1,2, \cdots, 50)$ to be considered, which are evaluated by five evaluation experts. Venture level is classified to three classes 1,2 and 3 . The bigger the value of venture level is, and the higher the venture of investment project is. Table 3 is an evaluation table about venture investment given by these five experts. The decision attribute $d$ determines a partition on the universe such that $U / \operatorname{IND}(d)=\left\{d_{1}, d_{2}\right\}=\{\{x \in$ $U: f(x, d)=$ Low $\},\{x \in U: f(x, d)=$ High $\}\}$.

Similar to Example 1, if each expert is corresponding to an equivalence relation, we obtain the global support factors, global certainty factors and global coverage factors for the "OR" and "AND" MGRS rules in Table 3 such that $\alpha^{\vee}=\alpha^{\wedge}=0.0168$, $\beta^{\vee}=\beta^{\wedge}=0.5, \gamma^{\vee}=\gamma^{\wedge}=0.0336$.

Following such computing results, we can see that

1. global support factor of "OR" MGRS rules = global support factor of "AND” MGRS rules;

2. global certainty factor of "OR" MGRS rules = global certainty factor of "AND" MGRS rules;

3. global coverage factor of "OR" MGRS rules = global coverage factor of "AND" MGRS rules. 
This is mainly because in Table 3, the computation of optimistic MGRS is same to that of pessimistic MGRS.

Table 3. An evaluation table about venture investment.

\begin{tabular}{ccccccc}
\hline$U$ & $E_{1}$ & $E_{2}$ & $E_{3}$ & $E_{4}$ & $E_{5}$ & $d$ \\
\hline$x_{1}$ & 2 & 3 & 3 & 2 & 3 & High \\
$x_{2}$ & 1 & 3 & 3 & 2 & 2 & High \\
$x_{3}$ & 1 & 1 & 1 & 1 & 1 & Low \\
$x_{4}$ & 1 & 1 & 1 & 1 & 1 & Low \\
$x_{5}$ & 1 & 1 & 1 & 1 & 1 & Low \\
$x_{6}$ & 2 & 2 & 1 & 2 & 2 & High \\
$x_{7}$ & 2 & 2 & 2 & 2 & 2 & Low \\
$x_{8}$ & 3 & 2 & 2 & 3 & 3 & High \\
$x_{9}$ & 2 & 3 & 2 & 3 & 1 & High \\
$x_{10}$ & 1 & 1 & 1 & 1 & 2 & Low \\
$x_{11}$ & 3 & 2 & 3 & 3 & 3 & High \\
$x_{12}$ & 2 & 3 & 3 & 2 & 3 & High \\
$x_{13}$ & 1 & 2 & 3 & 1 & 2 & Low \\
$x_{14}$ & 3 & 3 & 1 & 3 & 3 & High \\
$x_{15}$ & 2 & 1 & 1 & 1 & 1 & Low \\
$x_{16}$ & 2 & 2 & 2 & 2 & 1 & Low \\
$x_{17}$ & 2 & 1 & 2 & 2 & 2 & Low \\
$x_{18}$ & 1 & 1 & 3 & 1 & 2 & Low \\
$x_{19}$ & 3 & 3 & 3 & 3 & 3 & High \\
$x_{20}$ & 2 & 2 & 1 & 1 & 2 & High \\
$x_{21}$ & 2 & 2 & 2 & 1 & 1 & Low \\
$x_{22}$ & 1 & 3 & 1 & 1 & 2 & High \\
$x_{23}$ & 1 & 2 & 2 & 2 & 2 & High \\
$x_{24}$ & 1 & 1 & 2 & 2 & 1 & Low \\
$x_{25}$ & 1 & 1 & 2 & 2 & 2 & Low \\
$x_{26}$ & 1 & 3 & 1 & 2 & 2 & High \\
$x_{27}$ & 1 & 2 & 3 & 1 & 2 & Low \\
$x_{28}$ & 2 & 3 & 3 & 1 & 1 & High \\
$x_{29}$ & 2 & 3 & 3 & 2 & 1 & High \\
$x_{30}$ & 2 & 2 & 1 & 1 & 1 & High \\
$x_{31}$ & 2 & 3 & 1 & 1 & 1 & High \\
$x_{32}$ & 2 & 1 & 3 & 2 & 2 & Low \\
$x_{33}$ & 3 & 2 & 3 & 3 & 2 & High \\
$x_{34}$ & 1 & 1 & 3 & 1 & 1 & Low \\
$x_{35}$ & 2 & 2 & 2 & 1 & 1 & Low \\
$x_{36}$ & 2 & 2 & 2 & 1 & 1 & Low \\
$x_{37}$ & 2 & 3 & 2 & 2 & 2 & High \\
$x_{38}$ & 3 & 3 & 1 & 3 & 3 & High \\
$x_{39}$ & 1 & 1 & 1 & 1 & 2 & Low \\
$x_{40}$ & 3 & 3 & 1 & 3 & 3 & High \\
$x_{41}$ & 1 & 2 & 2 & 1 & 1 & Low \\
$x_{42}$ & 1 & 1 & 2 & 2 & 2 & Low \\
$x_{43}$ & 1 & 2 & 3 & 2 & 2 & Low \\
$x_{44}$ & 2 & 3 & 3 & 2 & 2 & High \\
$x_{45}$ & 3 & 3 & 2 & 3 & 3 & High \\
$x_{46}$ & 3 & 2 & 2 & 3 & 3 & High \\
$x_{47}$ & 2 & 1 & 1 & 1 & 1 & Low \\
$x_{48}$ & 2 & 1 & 1 & 1 & 2 & Low \\
$x_{49}$ & 3 & 3 & 2 & 3 & 3 & High \\
$x_{50}$ & 1 & 1 & 2 & 1 & 2 & Low \\
\hline & & & & & & \\
\hline
\end{tabular}

\section{Conclusions}

Measuring of decision rules play a crucial role in rough set based knowledge discovery. In this paper, the rules' measurements are firstly explored in terms of MGRS. Since MGRS may be considered as the compositions of multi-independent Pawlak's rough sets, the rules associated with MGRS are compositions of decision rules, which are derived from Pawlak's rough sets. In our approach, the support factors, certainty factors and coverage factors are proposed, respectively, to measure those MGRS rules. It should be noticed that such three factors are proposed in local and global views, respectively, which can be used to measure single MGRS rule and all of the MGRS rules in a decision system.

Since the measurements of MGRS rules have been proposed in this paper, then in our further researching, the multigranulation based decision performances in different decision systems are interesting topics to be addressed. For instance, the proposed local and global measurements can be generalized into incomplete decision systems ${ }^{26}$. In such case, a family of the binary relations instead of the equivalence relations will be used to constructed MGRS for analyzing MGRS rules.

Moreover, it should be noticed that since knowledge or attribute reduction is one of the key problems in rough set theory, then reducts of MGRS will help us to simplify the MGRS rules. The defined local and global measurements provide us theoretical basis for the definitions of reducts of MGRS.

\section{Acknowledgments}

This work is supported by the Natural Science Foundation of China (No. 61100116), Natural Science Foundation of Jiangsu Province of China (Nos. BK2011492, BK2012700), Natural Science Foundation of Jiangsu Higher Education Institutions of China (No. 11KJB520004), Postdoctoral Science Foundation of China (No. 20100481149), Postdoctoral Science Foundation of Jiangsu Province of China (No. 1101137C). 


\section{References}

1. Z. Pawlak, "Rough sets-theoretical aspects of reasoning about data," Kluwer Academic Publishers, (1992).

2. Z. Pawlak and A. Skowron, "Rudiments of rough sets," Inform. Sci., 177, 3-27 (2007).

3. Z. Pawlak and A. Skowron, "Rough sets: Some extensions," Inform. Sci., 177, 28-40 (2007).

4. Z. Pawlak and A. Skowron, "Rough sets and boolean reasoning," Inform. Sci., 177, 41-73 (2007).

5. D. Liu, T. R. Li, D. Ruan and W. L. Zou. "An incremental approach for inducing knowledge from dynamic information systems," Fund. Inform., 94, 245260 (2009).

6. D. Liu, T. R. Li, D. Ruan and J. B. Zhang. "Incremental learning optimization on knowledge discovery in dynamic business intelligent systems," J. Global Optim., 51, 325-344 (2011).

7. H. X. Li and X. Z. Zhou, "Risk decision making based on decision-theoretic rough set: a three-way view decision model," Int. J. Comput. Int. Syst., 4, 111 (2011).

8. D. Liu, Y. Y. Yao and T.R. Li, "Three-way investment decisions with decision-theoretic rough sets", Int. J. Comput. Int. Syst., 4, 66-74 (2011).

9. L. Zadeh, "Fuzzy logic equals computing with words", IEEE Trans. Fuzzy Syst. 4 103-111 (1996).

10. Y. Y. Yao, "Granular computing: past, present and future", 2008 IEEE International Conference on Granular Computing, 80-85 (2008).

11. T. Y. Lin, "Granular computing: practices, theories, and future directions", Encyclopedia on Complexity of Systems Science, 4339-4355 (2009).

12. Y. Y. Yao, "Triarchic theory of granular computing (in Chinese)", Quotient Space Theory and Granular Computing, Theory and Practice of Structured Problem Solving, Science Press, Beijing, 115-143 (2010).

13. Y. H. Chen and Y.Y. Yao, "A multiview approach for intelligent data analysis based on data operators", Inform. Sci., 178 1-20 (2008).

14. Y. H. Qian and J. Y. Liang, "Rough set method based on multi-granulations", 5th IEEE International Con- ference on Cognitive Informatics, 297-304 (2006).

15. Y. H. Qian, J. Y. Liang, Y. Y. Yao and C. Y. Dang, "MGRS: a multi-granulation rough set", Inform. Sci., 180, 949-970 (2010).

16. Y. H. Qian, J. Y. Liang and C. Y. Dang, "Incomplete multigranulation rough set", IEEE Trans. on Syst. Man Cy. A., 20, 420-431 (2010).

17. Y. H. Qian, J. Y. Liang and W. Wei, "Pessimistic rough decision", Second International Workshop on Rough Sets Theory, 440-449 (2010).

18. X. B. Yang, X. N. Song, H. L. Dou and J.Y. Yang, "Multi-granulation rough set: from crisp to fuzzy case", Annals of Fuzzy Mathematics and Informatic$s, 1,55-70$ (2011).

19. X. B. Yang, X. N. Song, Z. H. Chen and J. Y. Yang, "On multigranulation rough sets in incomplete information system," Int. J. Mach. Learn. Cyber., DOI 10.1007/s13042-011-0054-8.

20. W. H. Xu, Q. R. Wang and X. T. Zhang, "Multigranulation fuzzy rough sets in a fuzzy tolerance approximation space," Int. J. Fuzzy Syst., 13, 246-259 (2011).

21. H. M. Abu-Donia, "Multi knowledge based rough approximations and applications", Knowl.-Based Syst., 26, 20-29 (2012).

22. M. A. Khan and M. Banerjee, "Formal reasoning with rough sets in multiple-source approximation systems", Int. J. Approx. Reason. 49, 466-477 (2008).

23. W. Z. Wu and Y. Leung, "Theory and applications of granular labelled partitions in multi-scale decision tables", Inform. Sci., 181, 3878-3897 (2011).

24. Y. H. Qian, J. Y. Liang, W. Pedrycz and C. Y. Dang, "Positive approximation: an accelerator for attribute reduction in rough set theory", Artif. Intell., 174, 597618 (2010).

25. Y. H. Qian, J. Y. Liang, W. Pedrycz and C. Y. Dang, "An efficient accelerator for attribute reduction from incomplete data in rough set framework", Pattern Recognit., 44, 1658-167 (2011).

26. X. B. Yang and J. Y. Yang, "Incomplete information system and rough set theory: models and attribute reductions," Science Press \& Springer, (2012). 